\title{
Diabetes mellitus do tipo 2, síndrome metabólica e modificação no estilo de vida
}

\author{
Type 2 diabetes mellitus, metabolic \\ syndrome and change in lifestyle
}

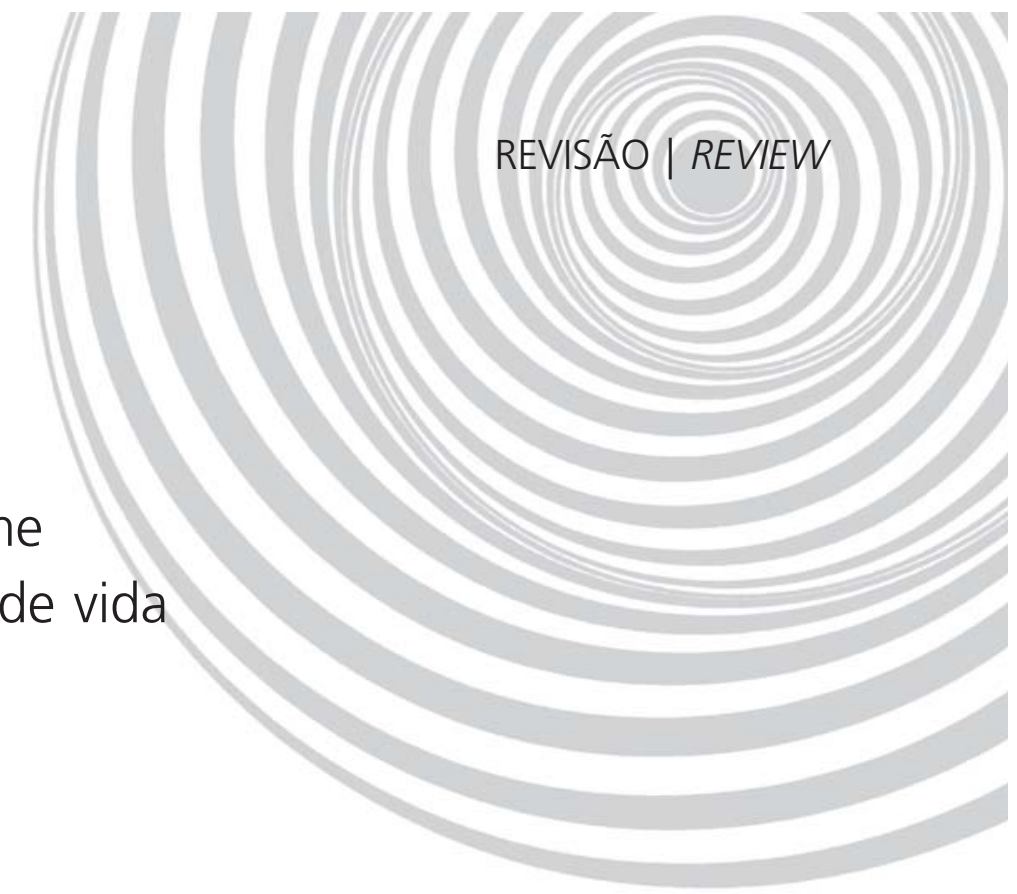

Kátia Cristina Portero McLELLAN ${ }^{1,2}$

Sandra Maria BARBALHO 3

Marino CATTALINI ${ }^{3}$

Antonio Carlos LERARIO'

\section{RE S U M O}

O Diabetes Mellitus do Tipo 2 favorece o aumento da morbidade e da mortalidade por doenças cardiovasculares. Essas doenças apresentam mesmo componente genético e mesmos antecedentes ambientais, sendo a resistência insulínica considerada um dos principais possíveis antecedentes. A síndrome metabólica é um transtorno complexo, representado por um conjunto de fatores de risco cardiovascular, usualmente relacionados à deposição central de gordura e à resistência à insulina. A modificação do comportamento alimentar inadequado e a perda ponderal, associadas à prática de atividade física regular, são consideradas terapias de primeira escolha para o tratamento da síndrome metabólica, por favorecer a redução da circunferência abdominal e da gordura visceral, melhorar a sensibilidade à insulina e diminuir as concentrações plasmáticas de glicose e triglicérides, aumentar os valores de HDL colesterol e, conseqüentemente, reduzir os fatores de risco para o desenvolvimento de Diabetes Mellitus do Tipo 2 e doenças cardiovasculares. Dessa forma, o presente artigo objetivou descrever e analisar alguns dos principais estudos publicados nas últimas décadas, os quais mostraram que a adoção de um estilo de vida adequado possibilita a prevenção primária do Diabetes Mellitus do Tipo 2. As mudanças no estilo de vida impróprio podem ser estimuladas por meio de uma intervenção educacional, dando ênfase ao aspecto nutricional e à atividade física, visando à redução dos fatores de risco relacionados à síndrome metabólica e às doenças cardiovasculares, em diferentes populações.

Termos de indexação: composição corporal; diabetes mellitus do tipo 2; estilo de vida; estudos de intervenção; síndrome x metabólica.

\section{A B S T R A C T}

Type 2 diabetes mellitus promotes increased morbidity and mortality from cardiovascular diseases. These diseases have the same genetic components and environmental antecedents and insulin resistance is considered

\footnotetext{
1 Universidade de São Paulo, Faculdade de Medicina, Hospital das Clínicas. São Paulo, SP, Brasil.

2 Pontifícia Universidade Católica de Campinas, Centro de Ciências da Vida, Faculdade de Nutrição. Av. John Boyd Dunlop, s/n., Jardim Ipaussurama, 13060-904, Campinas, SP, Brasil. Correspondência para/Correspondence to: K.C.P. McLELLAN. E-mail: <kaportero@yahoo.com.br>.

${ }^{3}$ Universidade Metodista de Piracicaba, Curso de Nutrição. Lins, SP, Brasil.
} 
516 | K.C.P. MCLELLAN et al.

one of the main possible antecedents. The metabolic syndrome is a complex disorder represented by a set of cardiovascular risk factors that are commonly associated with central adiposity and insulin resistance. Changing inadequate feeding habits, losing weight and exercising regularly are considered first-choice therapies in treating the metabolic syndrome since they reduce waist circumference, visceral fat and plasma concentrations of glucose and triglycerides; they improve insulin sensitivity and increase HDL cholesterol; consequently, they reduce the risk factors for type 2 diabetes mellitus and cardiovascular diseases. Thus, the objective of this article was to describe and analyze some of the main studies published in the last decades which showed that adopting a healthy lifestyle promotes the primary prevention of type 2 diabetes mellitus. An educational intervention that focuses on proper nutrition and exercise and, therefore, reduces the risk factors associated with metabolic syndrome and cardiovascular diseases, can help change inadequate lifestyles.

Indexing terms: body composition; diabetes mellitus, type 2; life style; intervention studies; metabolic syndrome.

\section{N T R O D U Ç Ã O}

O Diabetes Mellitus é uma síndrome de etiologia múltipla, decorrente da falta de insulina e/ou da incapacidade da mesma de exercer adequadamente seus efeitos, resultando em resistência insulínica. Caracteriza-se pela presença de hiperglicemia crônica, freqüentemente, acompanhada de dislipidemia, hipertensão arterial e disfunção endotelial'.

Essa enfermidade representa um considerável encargo econômico para o indivíduo e para a sociedade, especialmente quando mal controlada, sendo a maior parte dos custos diretos de seu tratamento relacionada às suas complicações, que comprometem a produtividade, a qualidade de vida e a sobrevida dos indivíduos, e que, muitas vezes, podem ser reduzidas, retardadas ou evitadas ${ }^{2,4}$. A progressiva ascensão das doenças crônicas, no Brasil, impõe a necessidade de uma revisão das práticas dos serviços de saúde pública, com a implantação de ações de saúde que incluam estratégias de redução de risco e controle dessas doenças ${ }^{5,6}$.

A educação em saúde, enquanto medida de prevenção ou retardo do Diabetes Mellitus, é uma ferramenta importante para a redução de custos para os serviços de saúde. As intervenções que focalizam aspectos múltiplos dos distúrbios metabólicos, incluindo a intolerância à glicose, a hipertensão arterial, a obesidade e a hiperlipidemia, poderão contribuir para a prevenção primária do Diabetes Mellitus ${ }^{7,8}$.
A modificação do comportamento alimentar inadequado e a perda ponderal, associadas à prática de atividade física regular, são consideradas terapias de primeira escolha para o tratamento da síndrome metabólica, por favorecer a redução da circunferência abdominal e da gordura visceral, melhorar a sensibilidade à insulina e diminuir as concentrações plasmáticas de glicose e triglicérides, aumentar os valores de HDL colesterol, e, conseqüentemente, reduzir os fatores de risco para o desenvolvimento de Diabetes Mellitus do tipo 2 e doença cardiovascular 9 .

O objetivo deste trabalho foi descrever e analisar alguns dos principais estudos publicados nas últimas décadas, os quais mostraram que a adoção de um estilo de vida adequado possibilita a prevenção primária do Diabetes Mellitus do tipo 2. As mudanças no estilo de vida impróprio podem ser estimuladas, dando ênfase ao aspecto nutricional e à atividade física, visando à redução dos fatores de risco relacionados à síndrome metabólica e às doenças cardiovasculares em diferentes populações.

\section{Diabetes Mellitus do Tipo 2}

O Diabetes Mellitus do tipo 2 favorece o aumento da morbidade e da mortalidade por doenças cardiovasculares. A íntima relação entre o Diabetes Mellitus do tipo 2 e as doenças cardiovasculares leva à hipótese do "solo comum", ou seja, as duas apresentam mesmo componente genético e mesmos antecedentes ambientais, sendo a resistência insulínica considerada um dos principais possíveis antecedentes ${ }^{10}$. 
Em publicação recente, Ciriello \& Motz ${ }^{10}$ apresentam uma revisão da hipótese do "solo comum", relacionando o stress oxidativo, a resistência à insulina, o Diabetes Mellitus e as doenças cardiovasculares. A Figura 1, adaptada da publicação original, exemplifica essa relação.

Ainda que o Diabetes Mellitus comprometa o metabolismo de todos os substratos energéticos, seu diagnóstico depende da identificação de alterações específicas da glicemia plasmática ${ }^{11}$. Na tolerância diminuída à glicose (IGT) e no
Diabetes Mellitus do tipo 2, se observa resistência à captação de glicose, estimulada pela insulina, independentemente da hiperglicemia, e a deterioração dessa tolerância dependerá da capacidade do pâncreas em manter o estado de hiperinsulinemia crônica. Entretanto, o fato de que um aumento na concentração plasmática de insulina poderia prevenir a descompensação da IGT, em um indivíduo insulino-resistente, não significa que esta resposta compensatória seja benigna. A resistência à captação de glicose, estimulada pela

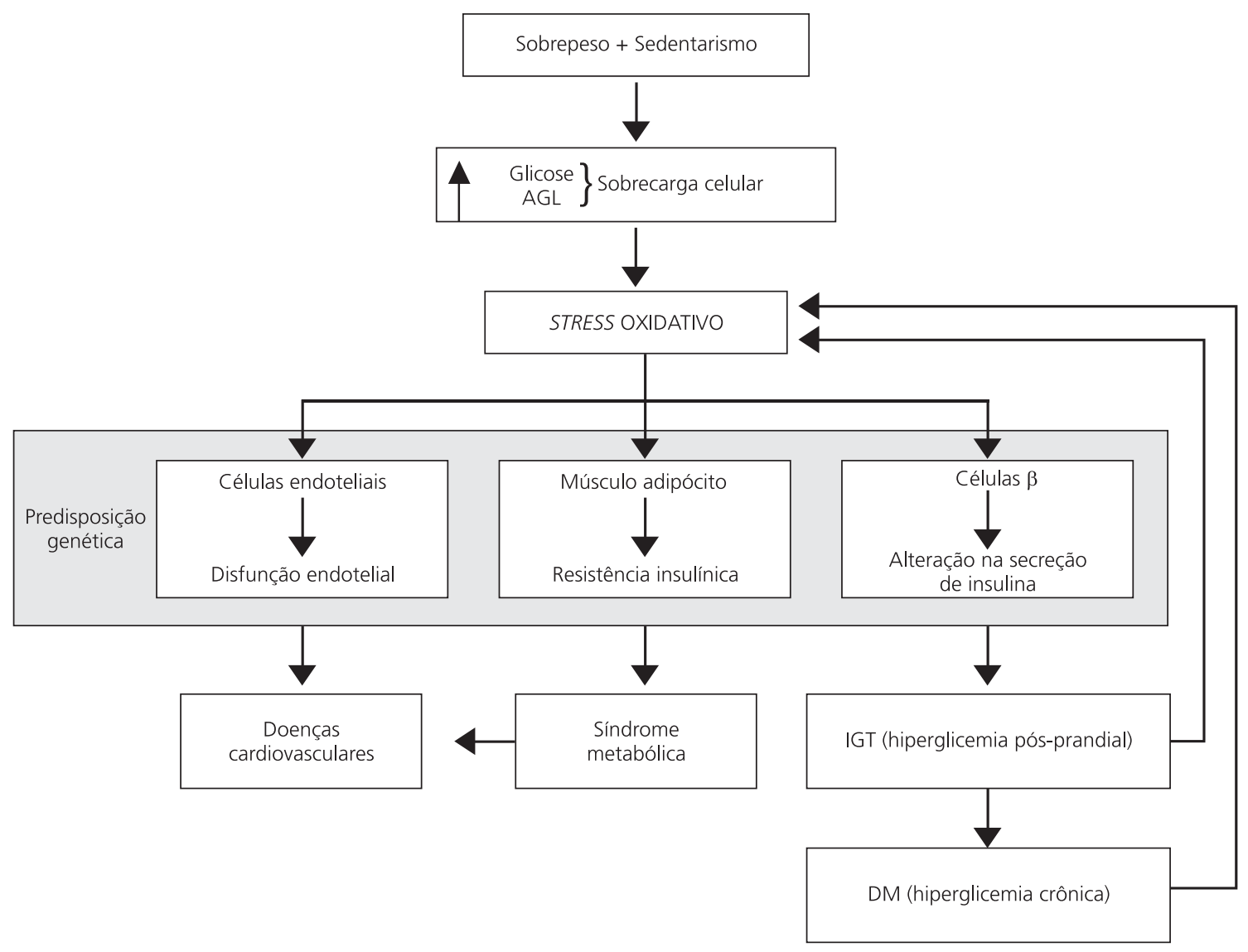

Figura 1. O sobrepeso e o sedentarismo levam ao aumento de glicose e ácidos graxos livres (AGL) nas células, que quando metabolizados e transformados em energia são acompanhados de um aumento na formação de radicais livres (stress oxidativo). As células musculares e os adipócitos podem se proteger desta condição, produzindo resistência à ação da insulina com o objetivo de reduzir a entrada de glicose e AGL nas células. As células $\beta$ e o endotélio são tecidos não dependentes de insulina, sendo assim a sobrecarga de glicose e AGL nestas células provoca stress oxidativo que induze a disfunção endotelial e das células $\beta$. A disfunção endotelial pode levar ao desenvolvimento de doenças cardiovasculares. A disfunção das células $\beta$ pode ser caracterizada por uma alteração na secreção de insulina. Esta condição se agrava na presença de resistência insulínica, visto que existe um maior requerimento secretório de insulina para a manutenção das concentrações plasmáticas normais de glicose. A disfunção precoce das células $\beta$, caracterizada por uma diminuição da secreção de insulina, e a hiperglicemia pós-prandial produzem o quadro clínico de tolerância diminuída à glicose (IGT). A hiperglicemia pós-prandial induz ao stress oxidativo. A persistência desta condição provoca exaustão das células $\beta$ e, conseqüentemente, o Diabetes Mellitus (DM). O stress oxidativo ocorre tanto na condição de IGT como de DM e pode contribuir para o desenvolvimento de doenças cardiovasculares. Além disso, todos os fatores de risco que acompanham a resistência à insulina também contribuem para o desenvolvimento de doenças cardiovasculares. 
518 K.C.P. MCLELLAN et al.

insulina, está associada a uma série de alterações que aumentam o risco para doenças cardiovasculares, intolerância à glicose, hiperinsulinemia, hipertrigliceridemia, redução do HDL-c, hipertensão arterial e obesidade andróide ${ }^{11}$.

As anormalidades na secreção de insulina podem levar ao desenvolvimento de resistência e, por outro lado, um comprometimento na captação de glicose pelos tecidos periféricos pode causar, secundariamente, falência das células $\beta$. As contribuições relativas da resistência à insulina e da deficiência de secreção das células $\beta$ para o desenvolvimento de hiperglicemia variam de paciente para paciente. A transição para o Diabetes Mellitus é determinada não somente por uma acentuação da resistência à insulina, atribuível ao excesso de peso e/ou envelhecimento, entre outras causas, como o sedentarismo, mas, também, pela incapacidade do pâncreas em aumentar a secreção insulínica adequadamente em resposta à hiperglicemia $^{11}$.

Na obesidade a secreção de insulina está aumentada, enquanto que a captação hepática e a eficácia periférica da insulina diminuem. A elevada secreção de insulina está relacionada ao grau de obesidade, já a redução na depuração hepática e a resistência periférica ao hormônio estão relacionadas ao tipo de obesidade (obesidade visceral). Os ácidos graxos livres aumentados na circulação, pela elevada sensibilidade lipolítica da gordura abdominal e pelo menor efeito antilipolítico da insulina nesse tecido, inibem a depuração hepática de insulina, levando à hiperinsulinemia e à resistência periférica, além do direcionamento desses ácidos graxos para a síntese de triglicérides pelo fígado ${ }^{11}$.

Os testes diagnósticos mais importantes para a identificação no distúrbio no metabolismo de carboidratos são, a glicemia de jejum e o teste oral de tolerância à glicose - oral glucose tolerance test (OGTT), sendo que este último informa tanto sobre a secreção de insulina como sobre sua ação periférica. Existe, ainda hoje, discordância sobre qual é o melhor teste preditivo para desenvolvimento do Diabetes Mellitus do tipo 2: glicemia de jejum entre $100-125 \mathrm{mg} / \mathrm{dL}$, que se caracteriza como glicemia de jejum alterada - impaired fasting glucose (IFG), ou glicemia de duas horas entre 140-199mg/dL, que é definida como tolerância diminuída à glicose - impaired glucose tolerance (IGT). IGT e IFG são duas classes distintas de alterações do metabolismo dos carboidratos. IGT detecta resistência com alteração precoce da secreção de insulina, tendo maior valor preditivo para doenças cardiovasculares, enquanto IFG reflete o distúrbio de secreção em uma fase mais tardia e está mais relacionada com a mortalidade por doença cardiovascular ${ }^{12}$.

Os critérios de diagnóstico do Diabetes Mellitus têm sido reavaliados, principalmente porque os pontos-limites da concentração da glicose plasmática, de valor maior ou igual a 126mg/ $\mathrm{dL}$, no jejum, e maior ou igual a $200 \mathrm{mg} / \mathrm{dL}$, em duas horas no OGTT, não são equivalentes para o diagnóstico de Diabetes Mellitus ${ }^{13}$. Quase todos os pacientes com concentrações glicêmicas no jejum acima de $140 \mathrm{mg} / \mathrm{dL}$, também têm valores de OGTT maiores ou iguais a $200 \mathrm{mg} / \mathrm{mL}^{14,15}$ e, pelo menos, um quarto dos indivíduos que têm OGTT $\geq 200 \mathrm{mg} / \mathrm{dL}$ apresentam glicemia de jejum $\geq 140 \mathrm{mg} / \mathrm{dL}$. Os OGTTs são, dificilmente, reproduzíveis, mais caros e pouco usados clinicamente para o diagnóstico do Diabetes Mellitus ${ }^{16}$. A Associação Americana de Diabetes (ADA) publicou, recentemente, valores mais baixos para o diagnóstico de glicemia de jejum alterada, que passaram de $110 \mathrm{mg} / \mathrm{dL}$ para $100 \mathrm{mg} / \mathrm{dL}^{12}$.

\section{Síndrome metabólica}

A síndrome metabólica (SM) é um transtorno complexo, representado por um conjunto de fatores de risco cardiovascular, usualmente, relacionados à deposição central de gordura e à resistência à insulina ${ }^{9}$. Essa síndrome foi descrita, pela primeira vez, por Reaven, em $1988^{17}$, e consistia na presença simultânea de vários fatores de risco cardiovasculares, como a hipertensão arterial (HAS), a intolerância à glicose, a hipertrigliceridemia e baixas concentrações de lipoproteína de alta densidade (HDL-c). 
A resistência à insulina (RI) refere-se à diminuição da ação da insulina endógena em seus tecidos-alvo, particularmente, músculos e tecido adiposo. Com o desenvolvimento da resistência, ocorre uma hiperinsulininemia compensatória, mas, com a evolução da doença, o indivíduo passa a apresentar deficiência na secreção de insulina, em função da exaustão da capacidade secretora das células $\beta$, o que culmina na sua incapacidade de manutenção das concentrações glicêmicas normais, no período pós-prandial. A essa fase de intolerância à glicose segue-se a hiperglicemia de jejum e a conseqüente instalação do quadro clínico de Diabetes Mellitus.

Recentes estudos indicam que o tecido adiposo apresenta capacidade secretora de substâncias com efeitos biológicos importantes com atuação, tanto local (efeitos parácrinos) como sistêmica (efeitos endócrinos), exibindo relação direta com a resistência à insulina. Essas substâncias são, na maioria, polipeptídeos, entre os quais se incluem: a leptina, a resistina, o peptídeo inibidor do ativador de plasminogênio (PAl-1), o fator de necrose tumoral (TNF- $\alpha$ ), a interleucina- 6 (IL-6), o peptídeo estimulador da acilação (ASP) e a adiponectina. Com exceção desta última, todos os demais são produzidos em maior quantidade com o aumento do tamanho do tecido adiposo. Ainda entre estes, a resistina, a IL-6, o TNF- $\alpha$ e a leptina estão envolvidos em processos geradores de resistência à insulina. A exceção é a adiponectina ${ }^{18}$, que atua aumentando a sensibilidade à insulina e cuja produção é diminuída com o aumento da massa adiposa ${ }^{19,21}$.

Existem diferentes fatores de risco associados à resistência à insulina e, portanto, à hiperglicemia, como é o caso da hipertensão arterial, das dislipidemias (aumento das taxas de LDL, colesterol total e triglicérides, e diminuição das taxas de HDL), da presença de um estado pró-coagulante (aumento de fibrinogênio e aumento do inibidor do ativador do plasminogênio) e da disfunção endotelial ${ }^{22}$.

\section{Modificação no estilo de vida}

O estilo de vida está diretamente relacionado com a incidência de Diabetes Mellitus do tipo 2 e da síndrome metabólica, e a obesidade e o sedentarismo aumentam dramaticamente esse risco. Alguns estudos mostraram que pessoas que consomem uma dieta rica em cereais integrais ${ }^{23,25}$ e ácidos graxos poliinsaturados ${ }^{26}$, associada ao consumo reduzido de ácidos graxos trans e de alimentos com elevado índice glicêmico ${ }^{23,24}$, apresentam riscos diminuídos para o desenvolvimento de Diabetes Mellitus.

O sedentarismo é um fator de risco para a obesidade tão importante quanto o consumo de dieta inadequada ${ }^{27}$, e possui uma relação direta e positiva com o aumento da incidência do Diabetes Mellitus do tipo 2 em adultos, independentemente do Índice de Massa Corporal (IMC) 28,29 ou de história familiar de Diabetes Mellitus ${ }^{30,31}$.

Vários estudos têm verificado os efeitos de intervenções na progressão de IGT para Diabetes Mellitus. Alguns estudos utilizaram, como estratégias de intervenção, medicamentos e dieta hipocalórica e balanceada ${ }^{32,33}$, dieta hipocalórica e balanceada e/ou exercício físico controlado ${ }^{34}$, ou a combinação de dieta hipocalórica e balanceada e exercício físico controlado, geralmente, referida como mudança no estilo de vida ${ }^{35-39}$ (Quadro 1).

O estudo finlandês de Tuomilehto et al. ${ }^{36}$, de prevenção do diabetes, foi a primeira pesquisa controlada que demonstrou que a prevenção é possível. A investigação teve uma duração média de 3,2 anos, e os 522 pacientes com IGT que participaram do estudo foram divididos em 2 grupos (controle e intervenção). Aos indivíduos do grupo controle, foram fornecidas orientações oral e escrita sobre alimentação saudável (folder de 2 páginas) e atividade física, no início do estudo e nas consultas anuais, porém nenhum programa individualizado e específico de mudança no estilo de vida foi oferecido a esse grupo. Os indivíduos do grupo de intervenção receberam orientação individual detalhada, oral e escrita, almejando 
$520 \mid$ K.C.P. MCLELLAN et al.

Quadro 1. Estudos de intenvenção na progressão do IGT para o DM.

\begin{tabular}{|c|c|c|c|c|}
\hline Autores & População & $\begin{array}{l}\text { Tempo de } \\
\text { estudo }\end{array}$ & Tipo de intervenção & Resultados obtidos \\
\hline Sartor et al. ${ }^{32}$ & $\begin{array}{c}\text { IGT } \\
(n=267)\end{array}$ & 10 anos & $\begin{array}{l}\text { Dieta hipocalórica e balanceada } \\
\text { e medicamento (Tolbutamida) }\end{array}$ & $\begin{array}{l}\text { O controle dietético, combinado com a } \\
\text { tolbutamida pode prevenir ou prolongar a } \\
\text { progressão do IGT para o DM. }\end{array}$ \\
\hline Chiasson et al. ${ }^{33}$ & $\begin{array}{c}\text { IGT } \\
(n=1.429)\end{array}$ & 3,3 anos & $\begin{array}{l}\text { Modificação no estilo de vida } \\
\text { Placebo e medicamento } \\
\text { (Acarbose) }\end{array}$ & $\begin{array}{l}\text { Acarbose pode ser utilizada como um trata- } \\
\text { mento alternativo ou em conjunto com a } \\
\text { modificação no estilo de vida para retardar } \\
\text { o desenvolvimento de DM2 em pacientes } \\
\text { com IGT. }\end{array}$ \\
\hline Pan et al. ${ }^{34}$ & $\begin{array}{c}\text { IGT } \\
(n=577)\end{array}$ & 6 anos & $\begin{array}{l}\text { Mudança no estilo de vida (dieta } \\
\text { hipocalórica + exercício físico com } \\
\text { duração, tipo e frequência con- } \\
\text { trolados) }\end{array}$ & $\begin{array}{l}\text { Os grupos: dieta, exercício físico e dieta + } \\
\text { exercício físico, estiveram associados à redu- } \\
\text { ção do risco para o desenvolvimento de DM } \\
\text { em } 31 \%, 46 \% \text { e } 42 \% \text {, respectivamente. }\end{array}$ \\
\hline Eriksson et al. ${ }^{35}$ & $\begin{array}{c}\text { IGT + DM } \\
(n=181+41)\end{array}$ & 6 anos & $\begin{array}{l}\text { Dieta hipocalórica e balanceada } \\
\text { e exercício físico controlado }\end{array}$ & $\begin{array}{l}\text { O programa de modificação no estilo de } \\
\text { vida mostrou melhoras substanciais no con- } \\
\text { trole metabólico e clínico em homens com } \\
\text { IGT e estágio primário de DM2. }\end{array}$ \\
\hline Tuomilehto et al. ${ }^{36}$ & $\begin{array}{c}\text { IGT } \\
(n=522)\end{array}$ & 3,2 anos & $\begin{array}{l}\text { Modificação do estilo de vida e } \\
\text { modificação intensa no estilo de } \\
\text { vida }\end{array}$ & $\begin{array}{l}\text { O risco de DM foi reduzido em } 58 \% \text { no } \\
\text { grupo de modificação intensa no estilo de } \\
\text { vida, sendo assim a incidência de DM esteve } \\
\text { diretamente relacionada a mudanças no } \\
\text { estilo de vida. }\end{array}$ \\
\hline Knowler et al. ${ }^{37}$ & $\begin{array}{c}\text { IGT } \\
(n=3.234)\end{array}$ & 2,8 anos & $\begin{array}{l}\text { Dieta hipocalórica e balanceada, } \\
\text { exercício físico e medicamento } \\
\text { (Metformina) }\end{array}$ & $\begin{array}{l}\text { A mudança no estilo de vida e o tratamento } \\
\text { com metformina reduziram a incidência de } \\
\text { DM, no entanto a mudança no estilo de } \\
\text { vida foi duas vezes mais eficaz na prevenção } \\
\text { do DM que a metformina. }\end{array}$ \\
\hline Bourn et al. ${ }^{38}$ & $\begin{array}{c}\text { IGT + DM } \\
(n=32+20)\end{array}$ & 2 anos & $\begin{array}{l}\text { Dieta hipocalórica e balanceada } \\
\text { e aumento na prática de exercícios } \\
\text { físicos }\end{array}$ & $\begin{array}{l}\text { Dieta e exercício físico resultaram na melho- } \\
\text { ra do controle metabólico e clínico de pes- } \\
\text { soas com IGT }\end{array}$ \\
\hline Torgerson et al. ${ }^{39}$ & $\begin{array}{c}\text { IGT } \\
(n=3.277)\end{array}$ & 4 anos & $\begin{array}{l}\text { Modificação no estilo de vida (di- } \\
\text { eta hipocalórica e balanceada e } \\
\text { exercícios físicos controlados) e } \\
\text { medicamento (Orlistat) }\end{array}$ & $\begin{array}{l}\text { A modificação no estilo de vida em conjun- } \\
\text { to com o uso de orlistat resultou em uma } \\
\text { maior redução na incidência de DM2 em } 4 \\
\text { anos e proporcionou uma maior redução } \\
\text { de peso. }\end{array}$ \\
\hline
\end{tabular}

IGT: tolerância à glicose diminuída; DM: Diabetes Mellitus.

uma redução no peso corporal de, pelo menos, $5 \%$, consumo de lipídeos inferior a 30\% do valor energético total (VET), consumo de ácidos gráxos saturados inferior a $10 \%$ do VET, aumento no consumo de fibras (pelo menos 15 gramas a cada mil calorias ingeridas) e incentivo à prática de atividade física (pelo menos 30 minutos/dia). Foi recomendado ao grupo de intervenção o consumo de cereais integrais, hortaliças, frutas, leite e derivados desnatados, carnes magras, óleos vegetais ricos em ácidos graxos monoinsaturados. Cada indivíduo do grupo de intervenção realizou
7 consultas com o nutricionista no primeiro ano, e uma consulta a cada três meses nos anos subseqüentes da pesquisa.

No grupo de intervenção, o risco de Diabetes Mellitus do tipo 2 foi reduzido em $58 \%$, em relação ao grupo de controle, e nenhum paciente que atingiu os 5 objetivos acima citados desenvolveu diabetes. Entre aqueles que não atingiram nenhum dos 5 objetivos, um terço se tornou diabético, comprovando que a redução no risco de desenvolver diabetes está ligada às mudanças do estilo de vida ${ }^{36}$. 
Para desenvolver e implementar programas de intervenção, é importante avaliar a importância relativa de mudanças nos diversos fatores relacionados ao estilo de vida, na alteração da tolerância à glicose e na incidência de Diabetes Mellitus, como dieta, peso corporal e atividade física. Hu et al. ${ }^{40}$ examinaram, simultaneamente, o padrão dietético e os fatores de estilo de vida relacionados ao risco para o desenvolvimento de
Diabetes Mellitus do tipo 2, de 84.941 mulheres, por um período de 16 anos, e mostraram que a modificação no estilo de vida (redução de peso, prática de exercícios físicos regulares, ingestão de alimentação equilibrada, abstinência do tabagismo e consumo limitado de bebidas alcoólicas) pode reduzir a progressão Diabetes Mellitus, tanto em pessoas de baixo como de alto risco.

Quadro 2. Estudos de investigação e intervenção referentes à composição dietética e/ou prática de atividade física.

\begin{tabular}{|c|c|c|c|c|}
\hline Autores & População & $\begin{array}{l}\text { Tempo de } \\
\text { estudo }\end{array}$ & Tipo de intervenção & Resultados obtidos \\
\hline Melin et al. ${ }^{21}$ & $\begin{array}{l}\text { Obesos } \\
(n=43)\end{array}$ & 2 anos & $\begin{array}{l}\text { Programa de modificação no es- } \\
\text { tilo de vida (dieta hipocalórica e } \\
\text { balanceada e exercício físico) em } \\
\text { dois grupos diferenciando-se na } \\
\text { intensidade }\end{array}$ & $\begin{array}{l}\text { Ambos os grupos apresentaram redução } \\
\text { de } 5 \text { - } 10 \% \text { do peso inicial. O grupo partici- } \\
\text { pante do programa intensivo de modifica- } \\
\text { ção no estilo de vida não apresentou uma } \\
\text { redução de peso estatisticamente maior } \\
\text { quando comparado com o outro grupo de } \\
\text { estudo. }\end{array}$ \\
\hline McAuley et al. ${ }^{42}$ & $\begin{array}{l}\text { Obesos com } \\
\text { RI }(n=79)\end{array}$ & 4 meses & $\begin{array}{l}\text { Programa de intervenção: dieta } \\
\text { hipocalórica balanceada (varian- } \\
\text { do na composição de lipídeos e } \\
\text { carboidratos) e exercício físico } \\
\text { com diferentes intensidades }\end{array}$ & $\begin{array}{l}\text { O grupo que participou do programa de } \\
\text { intevenção intensivo apresentou melhora na } \\
\text { sensibilidade à insulina. A melhora no con- } \\
\text { dicionamento aeróbio parece representar a } \\
\text { maior diferença entre os dois grupos de } \\
\text { estudo, embora a redução do peso corpo- } \\
\text { ral e a composição da dieta também desem- } \\
\text { penhem um papel importante na sensibili- } \\
\text { dade à insulina. }\end{array}$ \\
\hline Balkestein et al. ${ }^{43}$ & $\begin{array}{l}\text { Obesos } \\
(\mathrm{n}=37)\end{array}$ & 3 meses & $\begin{array}{l}\text { Dieta hipocalórica e balanceada } \\
\text { e exercício físico controlado }\end{array}$ & $\begin{array}{l}\text { A perda de peso aumentou a distensão da } \\
\text { artéria carótida, reduzindo a pressão arterial } \\
\text { e os exercícios físicos não resultaram em } \\
\text { efeitos adicionais no período do estudo. }\end{array}$ \\
\hline Lavrencic et al. ${ }^{44}$ & $\begin{array}{c}\text { SM } \\
(n=29)\end{array}$ & 3 meses & $\begin{array}{l}\text { Exercício físico controlado: tipo, } \\
\text { duração e frequência }\end{array}$ & $\begin{array}{l}\text { O programa de treinamento físico propor- } \\
\text { cionou efeitos positivos na função endotelial } \\
\text { de pacientes com SM, por meio da melhora } \\
\text { na dilatação mediada pelo fluxo da artéria } \\
\text { braquial. }\end{array}$ \\
\hline Hamdy et al. ${ }^{45}$ & $\begin{array}{l}\text { Obesos com } \\
\text { RI }(n=24)\end{array}$ & 6 meses & $\begin{array}{l}\text { Programa de modificação no es- } \\
\text { tilo de vida (dieta hipocalórica e } \\
\text { balanceada e exercício físico de } \\
\text { intensidade moderada) }\end{array}$ & $\begin{array}{l}\text { Melhora na função endotelial da artéria } \\
\text { braquial, redução da ativação vascular e im- } \\
\text { pacto positivo no sistema fibrinolítico de } \\
\text { pacientes obesos com SM independente do } \\
\text { grau de tolerância à glicose. }\end{array}$ \\
\hline Esposito et al. ${ }^{21}$ & $\begin{array}{l}\text { Obesas em } \\
\text { pré-menopausa } \\
\text { com HA ou } \\
\text { dislipidemia } \\
(\mathrm{n}=120)\end{array}$ & 2 anos & $\begin{array}{l}\text { Programa de modificação no es- } \\
\text { tilo de vida (dieta hipocalórica e } \\
\text { balanceada e exercício físico) di- } \\
\text { ferenciando-se no tipo de orien- } \\
\text { tação fornecida }\end{array}$ & $\begin{array}{l}\text { A perda de peso pode ser efetiva a longo } \\
\text { prazo por intermédio de uma abordagem } \\
\text { multidisciplinar de mudança no estilo de vida. } \\
\text { O programa mostrou melhora nos fatores } \\
\text { de risco cardiovascular no grupo de inter- } \\
\text { venção. O aumento no risco de desenvolvi- } \\
\text { mento de doenças cardiovasculares pode } \\
\text { ser associado, em parte, pelo aumento do } \\
\text { estímulo inflamatório (IL-6, IL-18 e PCR) e } \\
\text { pela redução dos mecanismos antiinfla- } \\
\text { matórios (adiponectina). }\end{array}$ \\
\hline
\end{tabular}

IL-6: interleucina 6; IL-18: interleucina 18; PCR: proteína C: reativa; HÁ: hipertensão arterial; RI: resistência à insulina; SM: síndrome metabólica. 
Alguns outros estudos foram realizados no intuito de avaliar a eficácia de programas de intervenção baseados nas recomendações tradicionais e intensivas, referentes à composição dietética e à prática de atividade física na redução de peso corporal $^{41}$, na melhora da sensibilidade à insulina ${ }^{42}$, na função endotelial e nos marcadores plasmáticos de ativação endotelial ${ }^{21}$ e coagulação $0^{43,45}$ (Quadro 2).

\section{CONSIDERAÇÕ ES FINAIS}

A incidência do Diabetes Mellitus do tipo 2 é crescente em nosso meio, e resulta da interação entre predisposição genética e fatores de risco ambientais e comportamentais. Ainda que a base genética do Diabetes Mellitus do tipo 2 não tenha sido identificada, há uma forte tendência a considerar que os fatores de risco modificáveis, como a obesidade e o sedentarismo sejam os determinantes não genéticos dessa enfermidade ${ }^{36}$.

A modificação no estilo de vida inadequado, o consumo de dieta equilibrada, associado à prática regular de atividade física, contribuem para o controle metabólico e a redução dos fatores de risco para a síndrome metabólica, o Diabetes Mellitus e as doenças crônicas não transmissíveis. Os estudos analisados neste artigo evidenciaram que o Diabetes Mellitus do tipo 2 pode ser prevenido em pacientes de alto risco, como os portadores de tolerância diminuida à glicose. Sendo assim, programas de intervenção que promovem mudança no estilo de vida devem ser incentivados, no intuito de melhorar a qualidade de vida da população de risco.

\section{REFERÊ N CIAS}

1. Sociedade Brasileira de Diabetes. Consenso Brasileiro sobre Diabetes 2002. Diagnóstico e classificação do diabetes melito e tratamento do diabetes melito tipo 2. São Paulo; 2003.

2. International Diabetes Federation. Diabetes health economics: facts, figures and Forecasts. Brussels: IDF; 1999.
3. Gross JL, Ferreira SRG, Franco L, Schmidt MI, Motta DG, Quintão E, et al. Diagnóstico e classificação do diabetes melito e tratamento do diabetes melito tipo 2. Recomendações da Sociedade Brasileira de Diabetes. Arq Bras Endocrinol Metabol. 2000; 44(4):Supl 1:8-35.

4. McLellan KCP, Motta DG, Lerario AC, Campino ACC. Custo do atendimento ambulatorial e gasto hospitalar do Diabetes Mellitus tipo 2. Saúde em Revista. 2006; 8(20):37-45.

5. Portero KCC, Motta DG, Campino, AAC. Abordagem econômica e fluxograma do atendimento a pessoas com Diabetes Mellitus tipo 2 na rede pública de saúde de um município paulista. Saúde em Revista. 2003; 5(11):35-42.

6. Portero KCC, Cattalini M. Mudança no estilo de vida para prevenção e tratamento do Diabetes Mellitus tipo 2. Saúde em Revista. 2005; 7(16): 63-9.

7. Franco LJ. Epidemiologia do Diabetes Mellitus. In: Lessa I. O adulto brasileiro e as doenças da modernidade: epidemiologia das doenças crônicas não transmissíveis. São Paulo: Hucitec; 1998. p.123-37.

8. American Diabetes Association. The prevention or delay of type 2 Diabetes. Diabetes Care. 2002; 25(4):742-9.

9. Sociedade Brasileira de Hipertensão, Sociedade Brasileira de Cardiologia. I Diretriz brasileira de diagnóstico e tratamento da síndrome metabólica. Arq Bras Cardiol. 2005; 84(Supl 1):1-28.

10. Ceriello A, Motz E. Is oxdative stress the patogenic mechanism underlying insulin resistance, diabetes, and cardiovascular disease? The common soil hypothesis revised. Arterioscler Thromb Vasc Biol. 2004; 24(5):816-23.

11. Wajchenberg BL, Santomauro ATMG, Santos RF. Diabetes Melito insulino-dependente (Tipo II): diagnóstico, etiopatogenia e fisiopatologia. In: Wajchenberg BL, organizador. Tratado de endocrinologia clínica. São Paulo: Roca; 1992. p.706-38.

12. American Diabetes Association. Follow-up report on the diagnosis of diabetes mellitus. Diabetes Care. 2003; 26(11):3160-7.

13. Report of the expert commitee on the diagnosis and classification of diabetes mellitus. Diabetes Care. 1997; 20(7):1183-97.

14. Harris MI, Hadden WC, Knowler WC, Bennett PH. Prevalence of diabetes and impaired glucose tolerance and plasma glucose levels in U.S. population aged 20-74 years. Diabetes. 1987; 36(4):523-34.

15. Peters $A L$, Davidson MB, Schriger DL, Hasselblad $\mathrm{V}$. A clinical approach for the diagnosis of diabetes 
mellitus: an analysis using glycosylated hemoglobin levels. Meta-analysis research group on the diagnosis of diabetes using glycated hemoglobin levels. JAMA. 1996; 276(15):1246-52.

16. The Expert Commitee on the Diagnosis on Classification of Diabetes Mellitus. Report of the expert commitee on the diagnosis on classification of diabetes mellitus. Diabetes Care. 2001; 24(Suppl 1):5-20.

17. Reaven GM. Banting Lecture: role of insulin resistance in human disease. Diabetes. 1988; 37(12): 1595-607.

18. Chandran M, Ciaraldi PSA, Henry R. Adiponectin: more than just another fat cell hormone? Diabetes Care. 2003; 26(8):2442-50.

19. Flier JS. The missing link with obesity? Nature. 2001; 409(6818):292-3.

20. Patane G, Anello M, Piro S, Vigneri R, Purrello F, Rabuazzo AM. Role of ATP production and uncoupling protein-2 in insulin secretory defect induced by chronic exposure to high glucose or free fatty acids and effects of PPARg inhibition. Diabetes. 2002; 51(9):2749-56.

21. Esposito K, Pontillo A, Palo CD, Giuliano G, Masella $M$, Marfella $R$, et al. Effect of weight loss and lifestyle changes on vascular inflammatory markers in obese women. JAMA. 2003; 289(14):1799-804.

22. Laaksonen DE, Lakka HM, Niskanen LK, Kaplan GA, Salonen JT, Lakka TA. Metabolic syndrome and development of diabetes mellitus: application and validation of resamply suggested definitions of the metabolic syndrome in a prospective cohort study. Am J Epidemiol. 2002; 156(11):1070-7.

23. Salmeron J, Manson JE, Stampfer MJ, Colditz GA, Wing AL, Willet WC. Dietary fiber, glycemic load, and risk of non-insulin-dependent-diabetes mellitus in women. JAMA. 1997; 277(20):472-7.

24. Salmeron J, Ascherio A, Rimm EB, Colditz GA, Spiegelman D, Jenkins DJ, et al. Dietary fiber, glycemic load and risk of NIDDM in men. Diabetes Care. 1997; 20(4):545-50.

25. Meyer KA, Kushi LH, Jacobs DR Jr, Slavin J, Sellers TA, Folsom AR. Carbohydrates, dietary fiber, and incidence of type 2 diabetes in older women. Am J Clin Nutr. 2000; 71(4):921-30.

26. Salmeron J, Hu FB, Manson JE, Stampfer MJ, Colditz GA, Rimm EB, et al. Dietary fat intake and risk of NIDDM in women. Am J Clin Nutr. 2001; 73(6): 1019-26.

27. Prentice AM, Jebb AS. Obesity in Britain: gluttony or sloth? BMJ. 1995; 311(7002):437-9.

28. Manson JE, Rimm EB, Stampfer MJ, Colditz GA, Willett WC, Krolewski AS, et al. Physical activity and incidence of non-insulin-dependent diabetes mellitus in women. Lancet. 1991; 338(8770): 774-8.

29. Hu FB, Willett WC, Li T, Stampfer MJ, Colditz GA, Manson JE. Adiposity as compared with physical activity in predicting mortality among women. N Eng J Med. 2004; 351(26):2694-703.

30. Zimmet PZ, McCarty DJ, Courten MP. The global epidemiology of non-insulin-dependent diabetes mellitus and the metabolic syndrome. J Diabetes Complications. 1997; 11(2):60-8.

31. Sartorelli DS, Franco LJ. Tendências do diabetes mellitus no Brasil: o papel da transição nutricional. Cad Saúde Pública. 2003; 19(Sup 1):29-36.

32. Sartor G, Schersten B, Carlstrom S, Melander A, Norden A, Persson G. Ten-year follow-up of subjects with impaired glucose tolerance: prevention of diabetes by tolbutamide and diet regulation. Diabetes. 1980; 29(1):41-9.

33. Chiasson JL, Josse RG, Gomis R, Hanefeld $M$, Karasik A, Laakso M. Acarbose for prevention of type 2 diabetes mellitus: the STOP-NIDDM randomised trial. Lancet. 2002; 359(9323): 2072-7.

34. Pan XR, Li GW, Wang WY, An ZX, Hu ZX, Lin J, et al. Effect of diet and exercise in preventing NIDDM in people with impaired glucose tolerance. The Da Qing IGT and Diabetes Study. Diabetes Care. 1997; 20(4):537-44.

35. Eriksson KF, Lindgärde R. Prevention of type 2 (non-insulin-dependent) diabetes mellitus by diet and physical exercise. The 6-year Malmö feasibility study. Diabetologia. 1991; 34(12):891-8.

36. Tuomilehto J, Lindström J, Eriksson JG, Valle TT, Hämäläinen $\mathrm{H}$, Ilanne-Parikka $\mathrm{P}$, et al. Prevention of type 2 diabetes mellitus by changes in lifestyle among subjects with impaired glucose tolerance. N Eng J Med. 2001; 344 (18):1343-50.

37. Knowler WC, Barret-Connor E, Fowler SF, Hamman RF, Lachin JM, Walker EA, et al. Reduction in the incidence of type 2 diabetes with lifestyle intervention or metformin. N Eng J Med. 2002; 346(6):393-403.

38. Bourn DM, Mann Jl, McSkimming BJ, Waldron MA, Wishart JD. Impaired glucose tolerance and NIDDM: does a lifestyle intervention program have an effect? Diabetes Care. 1994; 17(11):1311-9.

39. Torgerson JS, Hauptman J, Boldrin MN, Sjöström L. Xenical in the prevention of diabetes in obese subjects (XENDOS) study. Diabetes Care. 2004; 27(1):155-61.

40. Hu FB, Manson JE, Stampfer MJ, Colditz G, Lui S, Solomon $C$, et al. Diet, Lifestyle, and the risk of tipe 2 diabetes mellitus in woman. N Eng J Med. 2001; 345(11):790-7. 
41. Melin I, Karlström B, Lappalainen R, Berglund L, Mohsen $R$, Vessby B. A programme of behavior modification and nutritional counseling in the treatment of obesity: a randomized 2-y clinical trial. Int J Obes. 2003; 27(9):1127-35.

42. McAuley KA, Williams SM, Mann Jl, Goulding A, Chisholm A, Wilson N, et al. Intensive lifestyle changes are necessary to improve insulin sensitivity. Diabetes Care. 2002; 25(3):445-52.

43. Balkestein EJ, van Aggel-Leijssen DP, van Baak MA, Struijker-Boudier HA, van Bortel LM. The effect of weight loss with or without exercise training on large artery compliance in healthy obese men. Hypertension. 1999; 17:1831-5.
44. Lavrencic A, Salobir BG, Keber I. Physical training improves flow-mediated dilatation in patientes with the polymetabolic syndrome. Arterioscler Thromb Vasc Biol. 2000; 20(2):551-5.

45. Hamdy O, Ledbury S, Mullooly C, Jarema C, Porter $S$, Ovalle K, et al. Lifestyle modification improves endothelial function in obese subjects with the insulin resistance syndrome. Diabetes Care. 2003; 26(7):2119-25.

Recebido em: 21/3/2006

Versão final reapresentada em: 18/4/2007

Aprovado em: 11/6/2007 